\title{
Editorial
}

\section{New Candidate Species for Intensive Mediterranean Aquaculture: Studies on Reproduction, Digestion, Response to Stress}

This issue presents some articles concerning different features (feeding, reproduction, welfare and immune defence) which have been addressed during several research programs aimed at assessing the suitability of some fish species indicated as potential candidates for Mediterranean aquaculture diversification.

Over the last decade, finfish aquaculture production has been experiencing a worldwide expansion and is projected to become an increasingly significant source of animal proteins; it is estimated that, by 2015 , about $39 \%$ of world fish production will come from aquaculture. The current trend in European fish farming is to increase the production of marine species; however, the massive production of seabass (Dicentrarchus labrax) and gilthead seabream (Sparus aurata) in the Mediterranean area has resulted in the decline of economic profits, leading to the collapse of several fish farming operators. Therefore, Mediterranean aquaculture development is now at croassroads and new markets have to be found to ensure growth and viability to seafood industry. In this context, species diversification in aquaculture has been considered the most attractive strategy to expand actual market constraints and to open interesting perspectives of development in aquaculture productions.

The term "candidate species" conventionally indicates those species that could be of potential interest for aquaculture diversification as an alternative to traditionally farmed fish because of their high growth potential, good food conversion efficiency, easily adaptation to rearing and reproducing even at high density, and high market value. Some species have already been identified for this purpose and experimentations have started with fish belonging to the family Sparidae, such as the blackspot sea bream Pagellus bogaraveo, the sharpsnout sea bream Diplodus puntazzo and the common pandora Pagellus erythrinus, all common in western Mediterranean and eastern Atlantic, as well as to the family Carangidae, such as the greater amberjack, Seriola dumerilii.

However, the farming of new species requires many years of research and the main contraints which have to be addressed can be classified into:

(i) biological and technical aspects,

(ii) zootechnical constraints,

(iii) environmental concerns.

Research performed at the IAMC has focused on the suitability to farming of different species of high commercial value, using a multidisciplinary approach. Particularly, the following aspects of fish biology have been studied:

- the capability to adapt to rearing conditions;

- the parameters related to fish growth and nutrition (growth rates, food conversion ratio, feeding protocols, digestive enzymes);

- improvement of rearing protocols through manipulation of environmental factors, such as photoperiod;

- reproductive biology (sex ratio, reproductive strategies, gametogenesis and spawning in captivity);

- immune defence and fish health (non-specific immune parameters, response to stressors).

The present book is intended as a summary of knowledge acquired during a series of ten year-studies conducted on specimens reared in tanks at the facilities of IAMC Messina, and covers some issues until now scarcely investigated in the literature, i.e. reproduction and larval development, digestive physiology, the stress response, the non-specific immune system. 
Fish welfare is an important issue for aquaculture industry for production efficiency, quantity and product quality as well as for consumer perception and marketing. Strictly linked to fish welfare, another aspect concerns the study of non-specific immune parameters, since the relationship between immunity and maintenance of fish welfare has been established. A panel of selected parameters including cortisol, glucose, haemolysins, haemagglutinins and lysozyme, has been suggested as specific markers to investigate stress or altered health conditions.

Reproduction is one of those aspects of fish biology that are most critical to farming. In fact, many fish exhibit reproductive dysfunctions when reared in captivity, probably because of captivity-induced stress and/or the lack of appropriate "natural" spawning environment. Different aspects of sexuality, including hermaphroditism, gametogenesis, spermatozoal ultrastructure, maturity staging, seasonal development of gonads, and spawning in captivity, have been studied.

Other research concerns the physiology of the digestive system, the knowledge of which can contribute to solve the nutritional problems and assist in the success of farmed species. Massive production of these species is still limited because of hatchery problems with high mortalities occurring during larval rearing, particularly at transition from endogenous to exogenous feeding and weaning to artificial diets. Such problems, mainly arising from a lack of knowledge concerning nutritional requirements and feeding sequences, often result in low and unpredictable survival rates. Increasing knowledge of the digestive enzymes activities and nutritional requirements of the larvae of new candidate species for aquaculture could play a key role for development of optimal feeding protocols and greatly improve production under hatchery conditions. The ontogeny of the alimentary tract has been investigated in sharpsnout sea bream Diplodus puntazzo and common pandora Pagellus erytrinus and the differences have been analysed comparatively.

Attention has also been given to the suitability of plant-derived raw materials (i.e. Echium and linseed oils, Rice Protein Concentrate) as dietary components for the rearing of the blackspot sea bream, focusing on the effects of alternative plant dietary sources on growth performance, body composition and gastro-intestinal enzyme patterns in nutrient digestion of wild juveniles of this species.

I address this Special issue to all people interested in aquaculture, hoping that it could contribute to knowledge of the biology of some fish species considered as promising candidates in aquaculture; my sincere thanks are addressed to all the Authors who appreciated my project with enthusiasm.

\section{Dr. Gabriella Caruso (Guest Editor)}

National Research Council Istituto per l'Ambiente Marino Costiero

Spianata S. Raineri 86 - 98122 Messina, Italy

Tel: +39-090-669003

Fax: +39-090-669007

E-mail: gabriella.caruso@iamc.cnr.it

(C) Gabriella Caruso; Licensee Bentham Open.

This is an open access article licensed under the terms of the Creative Commons Attribution Non-Commercial License (http://creativecommons.org/licenses/by$\mathrm{nc} / 3.0 /$ ), which permits unrestricted, non-commercial use, distribution and reproduction in any medium, provided the work is properly cited. 